\title{
Beyond quasi-linear utility: strategy/false-name-proof multi-unit auction protocols (Short Paper)
}

\author{
Yuko Sakurai Yasumasa Saito Atsushi Iwasaki Makoto Yokoo \\ Graduate School of Information Science and Electrical Engineering \\ Kyushu University, Fukuoka, 819-0395 Japan \\ \{sakurai, saito\}@agent.is.kyushu-u.ac.jp \\ \{iwasaki, yokoo\}@is.kyushu-u.ac.jp
}

\begin{abstract}
We develop strategy/false-name-proof multi-unit auction protocols that can handle non-quasi-linear utilities.

One almost universal assumption in auction theory literature is that each bidder has quasi-linear utility, except for some works on budget-constrained bidders. In particular, the celebrated VCG protocol is strongly believed to critically depend on the quasi-linear assumption and will break down if this assumption does not hold. We show that with a simple modification, the VCG can handle non-quasi-linear utilities by sacrificing efficiency to a certain extent. The basic idea of this modification is that tentative allocation and payments are determined assuming quasi-linear utilities, but each bidder can choose the actual number of units to obtain based on his non-quasi-linear utility. The modified VCG only uses the gross utility of each bidder. Requiring gross utilities only is an advantage since collecting the entire utility function can be costly. However, determining tentative allocation and payments without considering actual non-quasi-linear utilities can cause significant efficiency loss. Furthermore, the VCG is not robust against false-name-proof.

Thus, we propose a new false-name-proof open ascending auction protocol in which each bidder declares his demand for a series of prices. This protocol can improve efficiency without collecting entire utility functions.
\end{abstract}

\section{Categories and Subject Descriptors}

I.2.11 [Artificial Intelligence]: Distributed Artificial Intelligence; K.4.4 [Computers and Society]: Electronic Commerce

\section{General Terms}

Economics, Theory

\section{Keywords}

Electronic Commerce, Auction mechanism design, Non-quasilinear utility, Strategy-proofness, False-name-proofness

Cite as: Beyond quasi-linear utility: strategy/false-name-proof multiunit auction protocols (Short Paper), Yuko Sakurai, Yasumasa Saito, Atsushi Iwasaki, and Makoto Yokoo, Proc. of 7th Int. Conf. on Autonomous Agents and Multiagent Systems (AAMAS 2008), Padgham, Parkes, Müller and Parsons (eds.), May, 12-16., 2008, Estoril, Portugal,pp.1609-1612.

Copyright (C) 2008, International Foundation for Autonomous Agents and Multiagent Systems (www.ifaamas.org). All rights reserved.

\section{INTRODUCTION}

Many theoretical/practical studies on Internet auctions have been conducted [3, 7]. One almost universal assumption of these works is that each bidder has quasi-linear utility, i.e., a bidder's utility is defined as the difference between his gross utility of allocated goods and his payment [5]. Here, gross utility means a bidder's utility for units when his payment is zero. One notable exception is works on budgetconstrained bidders in single-item/multi-unit auctions $[2,6]$. In these works, a bidder is assumed to have budget constraints, i.e., he cannot pay more than a predefined budget limit. His utility becomes minus infinity when his payment exceeds his limit. We can generalize such utilities to more general cases, such as piece-wise quasi-linear utility. For example, a bidder can pay up to certain amount from his own budget. If his payment exceeds that amount, he needs a loan and must pay interest. Such a utility can be represented as a separable utility function, where a bidder's utility is defined as the difference between his gross utility and an increasing function of his payment. In more general cases, we consider inseparable utility in which allocated goods and payment interact with each other and determine the utility. We can apply the well-known Vickrey-Clarke-Groves (VCG) protocol for multi-unit auctions. However, the VCG is strongly believed to critically depend on the quasi-linear assumption and will break down if this assumption does not hold. Borgs et al. showed the VCG is no longer strategy-proof if bidders have budget limits [2].

Furthermore, for Internet auctions, Yokoo et al. pointed out the possibility of a new type of fraud called false-name bids that exploit the anonymity available on the Internet [8, 11]. False-name bids are submitted under fictitious names, e.g., multiple e-mail addresses. Such deception is very difficult to detect, since identifying each participant on the Internet is virtually impossible. Although several false-nameproof multi-unit auction protocols have been developed, they also assume quasi-linear utilities.

We show a simple modification in which the VCG can handle non-quasi-linear utilities. The basic idea of this modification is that tentative allocation and payments are determined assuming quasi-linear utilities, but each bidder can choose the actual number of units to obtain based on his non-quasi-linear utility. More specifically, we utilize a general framework for describing strategy-proof protocols called the Price-Oriented Rationing-Free (PORF) protocol [9].

The modified VCG only uses the gross utility of each bid- 
der. Requiring only gross utility can be an advantage since collecting entire utility functions can be costly. However, determining tentative allocation and payments without considering actual non-quasi-linear utilities can cause significant efficiency loss. Furthemore, the VCG is not false-nameproof. Therefore, we develop a new false-name-proof, open ascending auction protocol based on an existing protocol called Ascending-price OPtion allocation protocol (AOP)[4]. The AOP was developed by modifying the Ausubel auction [1]. In this protocol, a bidder declares a demand for a series of prices announced by the auctioneer. For each announced price and for each bidder, the auctioneer calculates the aggregated demand of other bidders and allocates an option to a bidder to obtain the remaining units at the current price. When the total demand becomes less than the available units, a bidder can choose the optimal option among the allocated options. This can improve efficiency without collecting entire utility functions.

The rest of this paper is organized as follows. We describe the model of this problem setting (Section 2). Next, we explain a limitation of the VCG for budget-constrained bidders (Section 3). Then, with non-quasi-linear utilities, we describe how to modify the VCG to make it strategy-proof (Section 4). Furthermore, we describe our newly developed false-name-proof open ascending auction (Section 5).

\section{MODEL}

Assume $K$ units of homogeneous goods and a set of bidders $N=\{1,2, \ldots, n\}$ where $n \geq K$. Bidder $i$ determines his utility by privately observing a parameter or signal, $\theta_{i}$. We refer to $\theta_{i}$ as the type of bidder $i$ and assume $\theta_{i}$ is drawn from set $\Theta$. Let $u\left(\theta_{i}, k, p\right)$ denote the utility of the bidder with type $\theta_{i}$ when he obtains $k$ units and pays price $p$. We assume $u\left(\theta_{i}, 0,0\right)$ is normalized to 0 .

A utility function $u\left(\theta_{i}, k, p\right)$ is a quasi-linear utility if it is defined as the difference between the gross utility of the allocated goods and the payment: $u\left(\theta_{i}, k, p\right)=v\left(\theta_{i}, k\right)-p$. Here, $v\left(\theta_{i}, k\right)$ indicates bidder $i$ 's valuation of $k$ units and is identical to $u\left(\theta_{i}, k, 0\right)$.

Next, separable utility $u\left(\theta_{i}, k, p\right)$ is represented as the difference between gross utility and an increasing function of payment:

$$
u\left(\theta_{i}, k, p\right)=v\left(\theta_{i}, k\right)-f\left(\theta_{i}, p\right) .
$$

Here, we assume that $f$ is an increasing function of $p$ and is normalized at $f\left(\theta_{i}, 0\right)=0$. Without loss of generality, we assume $\forall p, f\left(\theta_{i}, p\right) \geq p$ holds $^{1}$. Utility with budget constraints and a piece-wise quasi-linear utility can be represented as special case of a separable utility function. Separable utility is a utility with budget constraints, if for a budget limit $b_{i}$,

$$
f\left(\theta_{i}, p\right)= \begin{cases}p, & p \leq b_{i} \\ \infty, & \text { otherwise }\end{cases}
$$

Separable utility is piece-wise quasi-linear if, for a series of budget limits $b_{i, 1}, b_{i, 2}, \ldots, b_{i, t}$,

$$
f\left(\theta_{i}, p\right)= \begin{cases}\alpha_{1} p, & p \leq b_{i, 1} \\ \alpha_{j} p, & b_{i, j-1} \leq p<b_{i, j} \\ \alpha_{t} p, & b_{i, t}<p\end{cases}
$$

Here, we assume $\alpha_{j} \geq 1$ for all $j$.

\footnotetext{
${ }^{1}$ If $f\left(\theta_{i}, p\right)<p$, we can change the monetary unit to satisfy this condition.
}

In the most general case, we can consider inseparable utility in which the allocated goods and the payment can interact with each other and determine the utility. For inseparable utility, we assume the following condition holds:

$$
\forall k, \forall p \leq p^{\prime} \quad u\left(\theta_{i}, k, p\right)-u\left(\theta_{i}, k, p^{\prime}\right) \geq p^{\prime}-p .
$$

This condition means that the utility decrease caused by the payment increase is at least (or more than) linear ${ }^{2}$.

Now, we introduce several properties that auction protocols should satisfy. An auction protocol is strategy-proof if for each bidder, declaring his true type is a dominant strategy, i.e., the optimal strategy for maximizing his utility regardless of other bidders' actions. An auction protocol is false-name-proof if for each bidder, declaring his true type using a single identifier, while he can use false-name bids, is a dominant strategy.

\section{LIMITATION OF VCG}

We define the VCG for $K$ units as follows. First, bidder $i$ declares his type $\theta_{i}$ to the auctioneer. Let us denote $\left(v\left(\theta_{i}, 1\right), \cdots, v\left(\theta_{i}, K\right)\right)$ as a valuation vector based on declared type. Then, the auctioneer determines an allocation and payments. For a set of feasible allocation $\mathbf{K}$ where $\mathbf{K}=$ $\left\{\mathbf{k}=\left(k_{1}, \cdots, k_{N}\right) \mid \sum_{i \in N} k_{i} \leq K\right\}$, we define $\mathbf{k}^{*}$ as an optimal allocation that maximizes the sum of the declared valuations in $\mathbf{K}$, formally, $\mathbf{k}^{*}=\arg \max _{\mathbf{k}} \sum_{i \in N} v\left(\theta_{i}, k_{i}\right)$. Aggregated utility function $V^{*}$ is defined as follows: $V^{*}\left(K, \Theta_{N}\right)=$ $\sum_{i \in N} v\left(\theta_{i}, k_{i}^{*}\right)$ where $k_{i}^{*} \in \mathbf{k}^{*}$. Then bidder $i$ 's price for $k_{i}^{*}$ units, $p_{i}\left(k_{i}^{*}\right)$, is calculated as follows:

$$
p_{i}\left(k_{i}^{*}\right)=V^{*}\left(K, \Theta_{N \backslash\{i\}}\right)-V^{*}\left(K-k_{i}^{*}, \Theta_{N \backslash\{i\}}\right) .
$$

Borgs et al. showed an example where the VCG does not satisfy strategy-proofness for bidders with budget constraints. In their example, bidder $i$ with budget limit $b_{i}$ is assumed to have linear gross utility, i.e., his utility for $k$ units is given as $k c_{i}$, where $c_{i}$ is a unit value. The VCG is applied to modified valuation vector $\left(v^{\prime}\left(\theta_{i}, 1\right), \cdots, v^{\prime}\left(\theta_{i}, K\right)\right)$ where $v^{\prime}\left(\theta_{i}, k\right)=\min \left(b_{i}, k c_{i}\right)$.

EXAMPle 1. Assume 2 bidders take part in an auction for 2 units. Suppose $b_{i}$ and $c_{i}$ as $\left(b_{1}, c_{1}\right)=(10,10)$ and $\left(b_{2}, c_{2}\right)=(5,3)$.

We compute the VCG payment using modified valuation vector $\left(v^{\prime}\left(\theta_{i}, 1\right), v^{\prime}\left(\theta_{i}, 2\right)\right)=\left(\min \left(b_{i}, c_{i}\right), \min \left(b_{i}, 2 c_{i}\right)\right)$. Bidders 1 and 2 's valuation vectors are calculated as $(10,10)$ and $(3,5)$. Thus, bidders 1 and 2 can obtain 1 unit at prices 2 and 0 , respectively. The utility of bidder 1 is calculated as $10-2=8$.

If bidder 1 declares his bids as $\left(b_{1}, c_{1}\right)=(10,5)$ by underbidding a value per unit, he can get 2 units at price 5 . As a result, bidder 1 's utility becomes $20-5=15$. This indicates that bidder 1 can increase his utility by underbidding.

\section{MODIFIED VCG}

We show that the VCG can handle non-quasi-linear utilities with simple modification.

\subsection{PORF protocol}

Describing a protocol as a PORF protocol simplifies proving that it is strategy /false-name-proof [9]. The following ${ }^{2}$ If this condition is not satisfied, we can change the mone-
tary unit to satisfy it. 
definition of a PORF protocol covers both cases of quasilinear and non-quasi-linear utilities. (1) bidder $i$ declares his type $\tilde{\theta}_{i}$ to the auctioneer. The declared type is not necessarily his true type $\theta_{i}$. (2) each price is defined for bidder $i$ and for any number of unit $k \leq K$. These prices must be determined independently of $i$ 's declared type, while it can be dependent on the declared types of other bidders. (3) for bidder $i$, the optimal number of units to maximize his utility is allocated. If multiple optimal allocations exist, one of them is determined.

\subsection{VCG for non-quasi-linear utility}

In quasi-linear cases, the VCG can be described as a PORF protocol. The VCG price of bidder $i$ for any $k$ units is determined as follows:

$$
p_{i}(k)=V^{*}\left(K, \Theta_{N \backslash\{i\}}\right)-V^{*}\left(K-k, \Theta_{N \backslash\{i\}}\right) .
$$

On the other hand, in non-quasi-linear cases, we redefine an aggregated utility function $V^{*}$ as follows:

$$
V^{*}\left(K, \Theta_{N}\right)=\max _{\mathbf{k} \in \mathbf{K}} \sum u\left(\theta_{i}, k_{i}, 0\right) .
$$

In the VCG for non-quasi-linear utilities, the auctioneer calculates a tentative allocation and the prices only based on the gross utility, i.e., applying formulas (6) and (7). Since the VCG is described as a PORF protocol, it is automatically strategy-proof. We only need to show that this protocol satisfies allocation feasibility.

First, we introduce one additional condition. Inseparable utility $u\left(\theta_{i}, k, p_{i}(k)\right)$ satisfies steepness condition if the following condition holds for all $k<k^{\prime}, p_{i}(k)$ :

$$
u\left(\theta_{i}, k, 0\right)-u\left(\theta_{i}, k, p_{i}(k)\right) \leq u\left(\theta_{i}, k^{\prime}, 0\right)-u\left(\theta_{i}, k^{\prime}, p_{i}(k)\right)
$$

This condition means that if the allocated number of units increases, the utility decrease caused by the price increase does not become smaller. For a separable utility function, this condition is automatically satisfied, since $u\left(\theta_{i}, k, 0\right)-$ $u\left(\theta_{i}, k, p_{i}(k)\right)$ is equal to $f\left(\theta_{i}, p_{i}(k)\right)$ for all $k$.

THEOREM 1. If bidders have non-quasi-linear utilities with the steepness condition, the modified VCG protocol satisfies allocation feasibility.

Proof. The outline of this proof is as follows. Assume bidder $i$ 's utility is maximized by obtaining $k$ units in quasilinear cases. Then for all $k^{\prime}>k$, the following condition must hold: $u\left(\theta_{i}, k, 0\right)-p_{i}(k) \geq u\left(\theta_{i}, k^{\prime}, 0\right)-p_{i}\left(k^{\prime}\right)$. From formulas (4), (8), and this above condition, we obtain $u\left(\theta_{i}, k, p_{i}(k)\right) \geq u\left(\theta_{i}, k^{\prime}, p_{i}\left(k^{\prime}\right)\right)$, i.e., bidder $i$ does not want to obtain $k^{\prime}$ units, where $k^{\prime}>k$.

EXAMPlE 2. We assume 2 bidders in an auction for 2 units, and bidders 1 and 2 's gross utility $\left(v\left(\theta_{i}, 1\right), v\left(\theta_{i}, 2\right)\right)$ are $(10,20)$ and $(1,2)$, respectively.

As a result, the VCG prices of bidder 1 are calculated as 1 for 1 unit and 2 for 2 units. The prices of bidder 2 are calculated as 10 for 1 unit and 20 for 2 units. Here, assume that bidders 1 and 2 have a budget limit of 10 and 8, respectively. Thus, while bidder 1 can obtain 1 unit at price 10 , bidder 2 can get no items because the price for 1 unit exceeds his budget.

\section{NEW OPEN ASCENDING AUCTION PRO- TOCOL}

We introduce a new false-name-proof multi-unit open ascending auction protocols for non-quasi-linear utilities.

\subsection{NQ-RSA}

First, we propose a false-name-proof sealed-bid auction in which a bidder declares a demand function, called the NonQuasi-linear Residual Supply Auction (NQ-RSA) protocol. In the NQ-RSA, we use a residual supply function facing each bidder to determine the prices. We define a demand function $d_{i}(c)$ of bidder $i$ to maximize his utility at unit price $c$. While a traditional definition of $d_{i}(c)$ assumes quasi-linear utilities, we generalize a demand function to a general case:

$$
d_{i}(c)=\inf \left\{k \mid \arg \max _{k} u\left(\theta_{i}, k, k c\right)\right\} .
$$

We define total demand $d(c)$ as the sum of $d_{i}(c)$ among a set of bidders $N: d(c)=\sum_{i \in N} d_{i}(c)$. Then, at price per unit $c$, the residual supply function facing bidder $i$ denoted by $s^{\sim i}(c)$, is defined as the difference between total supply $K$ and the number of units demanded by other bidders: ${ }_{s^{\sim i}}(c)=\max \left\{K-\sum_{l \neq i} d_{l}(c), 0\right\}$.

We can describe the NQ-RSA protocol as follows. (1) each bidder declares his type $\theta_{i}$. (2) the auctioneer determines the demand function based on declared type. Next, she generates a residual supply function and calculate a price per unit $c_{i}(k)$ for each $k$ units: $c_{i}(k)=\inf \left\{c \mid s^{\sim i}(c) \geq k\right\}$. (3) for bidder $i$, the optimal number of units $k_{i}^{*}$ is determined so that it maximizes the utility: $k_{i}^{*}=\arg \max _{k} u\left(\theta_{i}, k, k c_{i}(k)\right)$.

Example 3. Assume 2 units and 2 budget-constrained bidders. The minimum price unit is set to 0.1 . Bidders 1 and 2 's gross utility $\left(v\left(\theta_{i}, 1\right), v\left(\theta_{i}, 2\right)\right)$ are $(0,10)$ and $(6,12)$. Also, bidders 1 and 2 have a budget $b_{i}$ of 4 and 6 , respectively.

In this case, each bidder declares a demand function $d_{i}(c)$ as shown in Table 1.

\begin{tabular}{|c|c|c|c|c|c|}
\hline unit price $c$ & 0 & 2 & 2.1 & 3 & 3.1 \\
\hline$d_{1}(c)$ & 2 & 2 & 0 & 0 & 0 \\
\hline$d_{2}(c)$ & 2 & 2 & 2 & 2 & 0 \\
\hline$\sum_{i \in\{1,2\}} d_{i}(c)$ & 4 & 4 & 2 & 2 & 0 \\
\hline \hline$s^{\sim 1}(c)$ & 0 & 0 & 0 & 0 & 2 \\
\hline$s^{\sim 2}(c)$ & 0 & 0 & 2 & 2 & 2 \\
\hline
\end{tabular}

\section{Table 1: Situation of Example 3}

When the unit price is 2.1, bidder 2 's residual supply becomes 2 . Bidder 2 can get 2 units at unit price 2.1 .

Since the NQ-RSA is described as a PORF protocol, it automatically satisfies strategy-proofness. Thus, we need to show it satisfies allocation feasibility and false-name-proofness.

THEOREM 2. The NQ-RSA satisfies allocation feasibility, formally, $\sum_{i \in N} k_{i}^{*} \leq K$.

Proof. By assuming $\sum_{i \in N} k_{i}^{*}>K$, we derive a contradiction. We set $c_{t}$ to the minimum unit price among all $c_{i}$ where $k_{i}^{*}=d_{i}\left(c_{i}\right)$. For all bidders $i \neq t$, we get $d_{i}\left(c_{i}\right) \leq$ $d_{i}\left(c_{t}\right)$ based on $c_{i} \geq c_{t}$. Thus, we can obtain $d_{t}\left(c_{t}\right) \geq s^{\sim t}\left(c_{t}\right)$ based on $d_{t}\left(c_{t}\right)=k_{t}^{*}>K-\sum_{i \neq t} k_{i}^{*} \geq K-\sum_{i \neq t} d_{i}\left(c_{t}\right)$. This is inconsistent with the definition of $d_{t}\left(c_{t}\right)$. As a result, we can prove that $\sum_{i \in S} d_{i}\left(c_{i}\right)=\sum_{i \in S} k_{i}^{*} \leq K$. 
TheOREM 3. The NQ-RSA is false-name-proof.

Proof. We show that bidder $i$ cannot decrease his total payment even if the bidder uses two identifiers, $i^{\prime}$ and $i^{\prime \prime}$, compared with the payment when it uses one identifier, $i$.

Assume bidder $i^{\prime}$ can get $k^{\prime}$ units at unit price $c^{\prime}$ and bidder $i^{\prime \prime}$ can get $k^{\prime \prime}$ units at unit price $c^{\prime \prime}$. We also assume $c^{\prime} \leq c^{\prime \prime}$. By these assumptions, we can get $d_{i^{\prime}}\left(c^{\prime}\right) \leq K-$ $\sum_{l \neq i^{\prime}} d_{l}\left(c^{\prime}\right) \leq K-\sum_{l \neq\left\{i^{\prime}, i^{\prime \prime}\right\}} d_{l}\left(c^{\prime}\right)-d_{i^{\prime \prime}}\left(c^{\prime \prime}\right)$. As a result, we obtain $d_{i^{\prime}}\left(c^{\prime}\right)+d_{i^{\prime \prime}}\left(c^{\prime \prime}\right) \leq K-\sum_{l \neq\left\{i^{\prime}, i^{\prime \prime}\right\}} d_{l}\left(c^{\prime}\right) \leq s^{i}\left(c^{\prime}\right)$. We can prove bidder $i$ does not decrease his payment by submitting multiple bids.

\subsection{NQ-AOP}

We describe a new false-name-proof open ascending auction protocol for non-quasi-linear utilities, which is called the NQ-AOP. The NQ-AOP is developed by combining the techniques of the AOP protocol and NQ-RSA.

- The auctioneer announces unit price $c^{l}$ at a round $l \in$ $\{0, \ldots, L\}$. Then each bidder declares demand based on formula (9) at a current price.

Here, we set the conditions related to unit price and demand. First, the auctioneer cannot announce a lower price than the one called previously, i.e., $\forall l, c^{l-1}<c^{l}$. Next, bidder $i$ cannot declare higher demand than the one declared previously, i.e., $\forall c^{\prime}<c^{\prime \prime}, \quad d_{i}\left(c^{\prime \prime}\right) \leq d_{i}\left(c^{\prime}\right)$.

- The auction is closed at a round of $\mathrm{L}$ when satisfying $d\left(c^{L}\right) \leq K<d\left(c^{L-1}\right)$.

- Bidder $i$ can choose the optimal supply to maximize utility among a set of feasible supply $\left\{s_{i}^{l} \mid 1 \leq l \leq L\right\}$ :

$$
s_{i}^{l}=\min \left\{d_{i}\left(c^{l}\right), s^{\sim i}\left(c^{l}\right)\right\} .
$$

EXAMPLE 4. Let us consider a situation identical to Example 3. As described in Table 1, the auctioneer raises unit price $c^{l}$. At round $l$ when the auctioneer calls $c^{l}$, each bidder declares a demand $d_{i}\left(c^{l}\right)$. When the unit price reaches 2.1 , the aggregate demand is equal to 2 units. Thus, the auction ends at unit price 2.1 and the result is identical to Example 3.

THEOREM 4. In the NQ-AOP, truth-telling is a weakly dominant strategy for every bidder, when he can only know whether the auction is still open.

Proof. We prove that bidder $i$ cannot increase supply and improve his utility even if he declares $d_{i}^{\prime} \neq d_{i}\left(c^{l}\right)$.

When bidder $i$ under-declaring demand, i.e., $d_{i}^{\prime}<d_{i}\left(c^{l}\right)$, supply $s_{i}^{l}$ decreases by formula (10). On the other hand, when bidder $i$ over-declaring demand, i.e., $d_{i}^{\prime}>d_{i}\left(c^{l}\right)$, we consider two cases. If $d_{i}\left(c^{l}\right) \geq s^{\sim i}\left(c^{l}\right)$, bidder $i$ 's supply $s_{i}^{l}$ remains $s^{\sim i}\left(c^{l}\right)$ even if he over-declares a demand. If $d_{i}\left(c^{l}\right)<s^{\sim i}\left(c^{l}\right), d_{i}\left(c^{l}\right)$ is the optimal by formula (9). Thus, declaring $d_{i}^{\prime}$ cannot improve his utility.

THEOREM 5. In the NQ-AOP, truth-telling is an ex post perfect equilibrium even if every bidder can get any information about other bidders.

Due to space limitation, we omit the rigorous proof. The same argument for proving Theorem 4 can be applied to any strategy of other bidders, as long as the strategy does not react to bidder $i$ 's action.

We can prove that the NQ-AOP satisfies allocation feasibility and false-name-proofness by applying similar argument of Theorems 2 and 3, respectively.

\section{CONCLUSIONS}

We developed new strategy-proof/false-name-proof auction protocols when bidders' utilities are non-quasi-linear. Studies on auction mechanism design almost universally assume quasi-linear utility of each bidder. We demonstrated that with a simple modification, the VCG can handle nonquasi-linear utilities. Furthermore, we developed a new falsename-proof open ascending auction protocol.

Our future works include a more detailed theoretical/ experimental analysis of efficiency loss and developing falsename-proof combinatorial auction protocols in non-quasilinear utility cases.

\section{REFERENCES}

[1] L. Ausubel. An efficient ascending-bid auction for multiple objects. American Economic Review, 94(5):1452-1457, 2004.

[2] C. Borgs, J. Chayes, N. Immorlica, M. Mahdian, and A. Saberi. Multi-unit auctions with budget-constrained bidders. In Proceedings of the 6th ACM conference on Electronic commerce (EC'05), pages 44-51, 2005.

[3] P. Cramton, Y. Shoham, and R. Steinberg, editors. Combinatorial Auctions. The MIT Press, 2006.

[4] A. Iwasaki, M. Yokoo, and K. Terada. A robust open ascending-price multi-unit auction protocol against false-name bids. Decision Support Systems, 39(1):23-39, 2005.

[5] A. Mas-Colell, M. D. Whinston, and J. R. Green. Microeconomic Theory. Oxford University Press, 1995.

[6] E. S. Maskin. Auctions, development, and privatization: Efficient auctions with liquidity-constrained buyers. European Economic Review, 44:667-681, 2000.

[7] N. Nisan, T. Roughgarden, E. Tardos, and V. V. Vazirani, editors. Algorithmic Game Theory. Cambridge University Press, 2007.

[8] B. Rastegari, A. Condon, and K. Leyton-Brown. Revenue monotonicity in combinatorial auctions. In Proceedings of the 22nd Conference on Artificial Intelligence (AAAI-07), pages 122-127, 2007.

[9] M. Yokoo. The characterization of strategy/false-name proof combinatorial auction protocols: Price-oriented, rationing-free protocol. In Proceedings of the 18th International Joint Conference on Artificial Intelligence (IJCAI-03), pages 733-739, 2003.

[10] M. Yokoo, T. Matsutani, and A. Iwasaki. False-name-proof combinatorial auction protocol: Groves mechanism with submodular approximation. In Proceedings of the 5th international joint conference on Autonomous agents and multiagent systems (AAMAS '06), pages 1135-1142, 2006.

[11] M. Yokoo, Y. Sakurai, and S. Matsubara. The effect of false-name bids in combinatorial auctions: New fraud in internet auctions. Games and Economic Behavior, 46:174-188, 2004. 